\title{
La escolaridad en trabajadoras sexuales de la ciudad de San José, Costa Rica, al iniciarse el siglo XXI
}

\author{
Iris Amalia Ramírez Sánchez, Julián Monge-Nájera, Rosberly Rojas Campos y Roxana Morales Bonilla \\ Universidad Estatal a Distancia, 474 -2050 San Pedro Montes de Oca, San José, Costa Rica; iramirez@uned.ac.cr; julianmonge@gmail.com; \\ rrojas@uned.ac.cr; rmorales@uned.ac.cr
}

Recibido 3-VI-2009 Corregido 1-IV-2009 Aceptado 7-V-2009

\begin{abstract}
Schooling in sex workers from San José, Costa Rica, at the beginning of the twenty-first century. San Jose is the capitol city of Costa Rica, a small Central American country characterized by a higher educational level, higher per capita income and higher living standards than the rest of the countries in the region. A study in 1975 indicated that female sex workers in the city were women who had chosen sex work because they earned much more than in other job options available to women who only had finished part of the primary school, and that nearly half did not want to change their activity. In order to know whether the situation had changed three decades later, in 2008 we interviewed 78 women who perform sex work in the city, with fares between US\$3 and US\$100 per hour and ages between 18 and 81 years. The interviews were based on a questionnaire; respondents were paid for their time, and data were analyzed with the SPSS statistical program. Most of these workers are Costa Ricans of urban origin, few have education beyond primary school, and they consider that the school years that they approved -despite receiving little help in this area from their families- are among their main achievements in life. One third of the women said that they had had a pleasant childhood, and most stated that they do not see a conflict between their work and religious beliefs because they provide sexual services to support their children (most are single mothers). Some believe that their work reduces sexual violence; most use condoms as protection and believe in the patriarchal view that women raise families while men provide resources. Universities could support these women with grants and advice; and finance more studies to separate myth from reality in this field, for example, with studies based on life histories, a technique that would give sex workers a voice from the conception of their own worlds and the environment in which they develop.
\end{abstract}

\section{KEY WORDS}

Education, sex work, socialization, patriarchy, culture, prostitution.

La ciudad conurbada de San José, en Costa Rica, tenía en 1980 unos 730000 habitantes (Costa Rica Ministerio de Economía 1998), de los cuales unas 1400 eran mujeres dedicadas al trabajo sexual en aproximadamente 200 lugares de la ciudad (Acuña et al. 1982). Esto corresponde a un índice de 2 trabajadoras sexuales por cada 1000 habitantes. Uno de los primeros estudios que incluyó el tema de la escolaridad encontró que en el San José de la década de 1970 estas trabajadoras eran mujeres que habían elegido el trabajo sexual porque ganaban mucho más que en las demás opciones laborales que tenían, dado su nivel de escolaridad, en general limitado a la escuela primaria, y que cerca de la mitad no deseaban cambiar de actividad (Vega 1975).

Una encuesta hecha en 1980 a 348 mujeres halló que eran en general madres solteras o separadas de sus maridos, con una media de 2,6 hijos por mujer; y que un $70 \%$ inició el trabajo sexual siendo mayores de edad, muchas tras haber tenido otros trabajos. Un $68 \%$ declararon no haber tenido problemas familiares importantes, y aunque el $80 \%$ tenía la educación primaria concluida, muy pocas tenían nivel universitario. La diferencia de tarifa por sus servicios era de 50 a 300 colones por cliente (siendo muchos de sus clientes hombres casados), el $60 \%$ tenía clientes fijos con los cuales mantenía una relación amistosa o incluso sentimental, y un 45\% no deseaba cambiar de trabajo (Acuña et al. 1982).

Al iniciarse la década de 1990, una entrevista a 32 trabajadoras encontró que el ámbito de edad era 29-32 años, 
que el $75 \%$ eran mujeres sin pareja pero con hijos que mantener, y que como regla sus hermanas no se dedicaron al trabajo sexual a pesar de tener el mismo historial de abuso sicológico, físico y sexual de parte de mujeres y hombres de su propia familia. También declararon tener baja autoestima; un deseo de ser esposas y madres; relaciones emocionales estables con clientes fijos y un cambio hacia el uso sistemático del preservativo por miedo al sida. En general eran mujeres provenientes de familias de bajos ingresos y tenían educación secundaria incompleta (Ortiz et al. 1998).

Históricamente, las trabajadoras sexuales han sido vistas en Costa Rica como seres desviados, como enemigas de la familia y de la moral cristiana. Antes de 1949 el trabajo sexual era ilegal, lo que permitía que estas mujeres fueran violadas y explotadas por la policía y generó la aparición de "protectores" ("chulos") (Marín 2005). La abolición de esa ley eliminó casi totalmente esas violaciones y a los chulos. Al iniciarse el siglo XXI, la derecha cristiana está intentando volver a la legislación prohibicionista abolida en 1949 y los medios de comunicación crean un ambiente amarillista sobre turismo sexual (Marín 2005).

La persecusión institucional y social de las trabajadoras sexuales, y en menor grado, de sus clientes, tiene sus orígenes en el hecho de que la educación en Costa Rica, al igual que en la mayoría de países del mundo, transmite a cada generación los valores y tradiciones que conforman la ideología patriarcal, aun cuando públicamente se afirme justo lo contrario (Ramírez et al. 2005).

Las trabajadoras sexuales están en esa actividad porque en ella ganan mucho más dinero que en las otras opciones que tienen, dada su formación escolar, por lo que no desean cambiar de actividad (Ortiz et al. 1998).

Este estudio describe y analiza la relación que han tenido con la educación las trabajadoras sexuales del centro de la ciudad de San José, Costa Rica, a lo largo de sus vidas, y sugiere cómo la educación a distancia puede proveerles un aumento en su nivel de escolaridad.

\section{METODOLOGÍA}

Definimos trabajo sexual como una transacción comercial en que el cliente paga por actividad sexual y entrevistamos a 78 mujeres que lo realizan en la ciudad de San José, capital de Costa Rica.

\section{Muestra}

Las personas entrevistadas corresponden a tres grupos cuyas características se definen a continuación:

\section{Grupo 1: Ambulantes}

Mujeres que trabajan en la zona aledaña al Mercado Borbón y Mercado Central, encuentran a sus clientes en la calle y su tarifa oscila entre los US\$3 y los US\$8 por hora. A este grupo pertenecen las entrevistadas de mayor edad (26-81 años). Entrevistamos a diez que frecuentan La Sala, lugar donde descansan, comparten socialmente y reciben apoyo para conocer y defender sus derechos. Allí se realiza trabajo comunal de la Universidad de Costa Rica y visitas del Ministerio de Salud. Se trata de mujeres físicamente menos atractivas y con bajo nivel de escolaridad.

También entrevistamos a diez mujeres de la Asociación RAHAB, un grupo religioso privado que les ofrece alimentos y cursos, a cambio de participar en actividades de su organización religiosa y con el requisito de dejar el trabajo sexual en un periodo definido a partir de su entrada a la asociación (RAHAB 2008). Predominan las mujeres físicamente deterioradas y afectdas por el ambiente, que están tratando de dejar el trabajo sexual y con bajo nivel de escolaridad.

\section{Grupo 2: De Sala}

Trabajadoras de dos salas de masajes de San Juan de Tibás. Sus tarifas oscilan de US\$18 a US\$72 por hora y sus edades de 18 a 33 años. Son mujeres con un nivel de escolaridad intermedio.

\section{Grupo 3: Independientes o VIP}

Trabajadoras independientes oVIP, que cobran US\$100 la hora y tienen entre 19 y 38 años de edad. Consiguen a sus clientes mediante visitas a bares (especialmente en la zona cercana al Parque Morazán) y por medio de Internet. A menudo tienen clientes fijos, a veces durante años, y establecen una relación más estable. Son mujeres de apariencia física muy atractiva y educación que generalmente incluye la secundaria y en algunos casos, universitaria.

\section{Método}

Durante 2007 entrevistamos a 58 mujeres usando el cuestionario del Apéndice 1. El cuestionario tiene tres ejes temáticos:

- La trabajadora: su pasado, presente y futuro.

- El cliente: quién es, qué pide y cómo la trata.

- El dinero (cuánto es y cómo se distribuye) y la sociedad (qué le dio, qué le pide y cómo la trata).

Estos datos se complementaron con 20 entrevistas hechas entre 2004 y 2006 en las salas de masaje de Tibás, con una versión anterior del cuestionario que difería 
únicamente en que tenía menos preguntas por eje.

El equipo de investigación es multidisciplinario (biología, economía, sociología y trabajo social) y partió de los siguientes principios:

1. Enfoque científico sobre el fenómeno a estudiar.

2. No juzgar subjetivamente el trabajo sexual como malo o bueno.

3. Respetar la opinión de quienes lo ejercen, opinión tradicionalmente desechada por la sociedad y los mismos estudiosos del tema.

4. Evitar simplificaciones acientíficas, de un fenómeno que es muy complejo e incluye desde mujeres que realizan los servicios sexuales por necesidades básicas, hasta quienes trabajan al más alto nivel por deseo o incluso por convicción.

Una consulta preliminar a varias salas de masaje demostró que no había disposición a participar en el estudio, por lo que descartamos la idea original de un muestreo al azar. En La Sala, RAHAB y las dos salas de masajes, hubo disposición a participar. Para muestrear al grupo de mujeres que trabajan en forma independiente, usamos el método de "bola de nieve". Esto lo hicimos mediante la intermediación de una trabajadora independiente que estaba haciendo un periodo en una sala de masaje debido a que sus clientes fijos estaban fuera de Costa Rica. Ella trajo a varias amigas quienes a su vez trajeron para las entrevistas a sus conocidas que trabajan en este campo. Este método ha demostrado previamente su viabilidad, validez estadística y representatividad para estudiar temas de sexualidad, trabajo sexual y conducta privada en Costa Rica (Gutiérrez 1994).

Por el tiempo dedicado a la entrevista (aproximadamente una hora), cada mujer recibió un pago en efectivo igual al que habría recibido si hubiera atendido a un cliente. La información obtenida de las participantes se dio en un ambiente individual, en donde se les proporcionó un espacio de seguridad y de confianza favorecido por el uso de un seudónimo para cada una.

Hicimos "triangulación" (comparación crítica de información) entrevistando de manera separada a cerca de 20 mujeres de cada grupo y comparando sus respuestas. También comparamos lo que dijeron, con dos entrevistas abiertas a personas que alquilaban las habitaciones a las trabajadoras de Tibás. En tres casos, se entrevistó de nuevo a la misma persona con varios meses de diferencia para comparar las respuestas.

También hicimos dos grupos focales en los cuales discutimos los resultados preliminares con media docena de las entrevistadas en las salas de masaje.

\section{RESULTADOS}

\section{Quienes son}

\section{Lugar de procedencia}

La mayoría (58\%) son de origen urbano (Fig. 1). Las inmigrantes rurales son menos. Por ejemplo, María del grupo 1, dijo "nací en Puriscal y fui abandona en un caño por mi madre biológica y criada por mi madrina. Viví allí hasta los 15 años y me junté con un hombre alcohólico que me maltrataba y me trajo a vivir a Guadalupe, que es el lugar en que más me ha gustado vivir".

Sandra, de 43 años, relató que nació en Sixaola de Limón, en donde vivió hasta los 12 años, edad en que su familia la entregó a una conocida, quién se la trajo a San José; luego fue abandonada, y aun añora su pueblo natal.

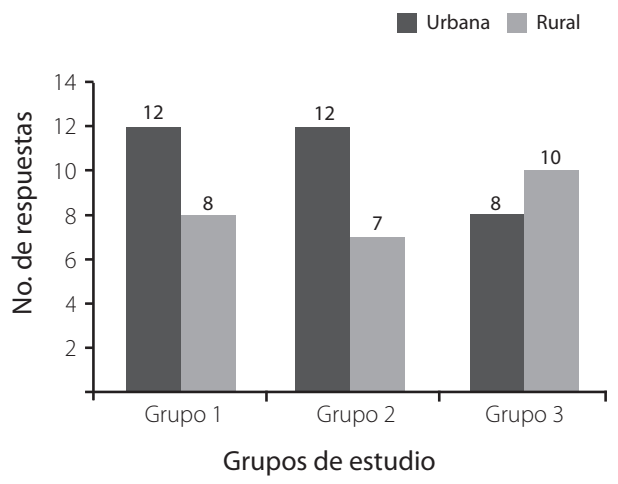

FIG. 1. Lugar de procedencia de las trabajadoras sexuales entrevistadas.

\section{Su historia}

\section{Nivel educativo y apoyo durante los años de formación}

La mayoría de estas mujeres concluyó la educación primaria, muy pocas tienen nivel universitario. María de 50 años y participante en el grupo 1, refiere que llegó hasta primer grado. La sacaron de la escuela y no pudo estudiar, sabe leer y firmar, pero no escribir.

Las mujeres de mayor edad tienden a tener escolaridad más baja que las jóvenes (Coeficiente de correlación $r=-0.3805 ; p=0.0042$ ).

En los tres grupos, la mayoría de las mujeres indicaron que de niñas no recibían ayuda en su casa para elaborar las tareas escolares y estudiar (Cuadro 1). Elvira de 49 años y del grupo 1, afirma que llegó hasta el cuarto grado de escuela, pero tuvo que dejarla por problemas de salud y 


\section{CUADRO 1}

Respuestas de las entrevistadas a la pregunta ¿Quién le ayudaba con las tareas escolares?

\begin{tabular}{lccc}
\hline \multicolumn{1}{c}{ Familiares } & Grupo 1 & Grupo 2 & Grupo 3 \\
\hline Nadie & 9 & 6 & 7 \\
Madre & 1 & 4 & 7 \\
Padre & 0 & 0 & 1 \\
Ambos padres & 1 & 6 & 1 \\
Otros* $^{*}$ & 4 & 2 & 1 \\
NR & 6 & 2 & 0 \\
\hline
\end{tabular}

*Primos, abuelos o hermanos.

que nunca nadie le ayudó en las tareas.

El segundo grupo en tamaño es el de quienes eran ayudadas por sus madres, como señala Lucy, de 19 años con estudios universitarios y participante del tercer grupo: "Mi mamá me apoyaba con el inglés que a mí no me gusta. En matemáticas generales salgo mal, tal vez desde que un profesor de matemáticas en el colegio me dijo que yo era una burra, me enojó tanto que le tiré el borrador, pero en cambio en contabilidad y cálculo que me gusta me iba super bien".

El apoyo provino en menor parte de otros familiares, como abuelos, hermanos y otros; Paola de 24 años manifiesta: "mi padre le daba pensión a mi abuela para que yo estudiara, ella me ayudaba con el estudio y mi prima, que es tres años mayor, también me ayudaba a veces".

\section{Relaciones de género en la niñez}

En general, de niñas jugaban con otras niñas y con niños (Cuadro 2). Seis mujeres del grupo 2 afirmaron que no jugaban y por diversos motivos no socializaban, al respecto Marisol externa: "en mi niñez no jugué, recuerdo que solo un diciembre mi mamá me regaló una muñeca

\section{CUADRO 2}

Respuestas de las entrevistadas a la pregunta ¿Jugaba más con niñas, niños o con ambos sin preferencias?

\begin{tabular}{lccc}
\hline $\begin{array}{c}\text { Compañeros (as) } \\
\text { de juego }\end{array}$ & Grupo 1 & Grupo 2 & Grupo 3 \\
\hline Niñas & 9 & 6 & 7 \\
Niños & 1 & 4 & 7 \\
Con ambos & 0 & 0 & 1 \\
No jugaba & 1 & 6 & 1 \\
NR & 6 & 2 & 0 \\
\hline
\end{tabular}

de cartón, a mis hermanos papá se los llevaba a trabajar en construcción".

\section{Principales logros en la edad preadulta}

En los tres grupos se dio una tendencia a señalar los estudios como el principal logro, aunque no los concluyeran. El trabajo y otros aspectos casi no fueron mencionados.

Sin embargo, el trabajo si constituye un logro deseado pero no alcanzado. En general las entrevistadas no tienen formación para realizar un trabajo más calificado y solo una minoría labora en otras actividades (Fig. 2). Algunas del grupo 1 refieren hacer trabajo doméstico por horas, recolección de deshechos para reciclar y ventas ambulantes. En el grupo 2 una que está en proceso de retirarse del trabajo sexual gana algún dinero limpiando casas, vende ropa interior por catálogo y también objetos para cocina, alfombras y otros por pedido.

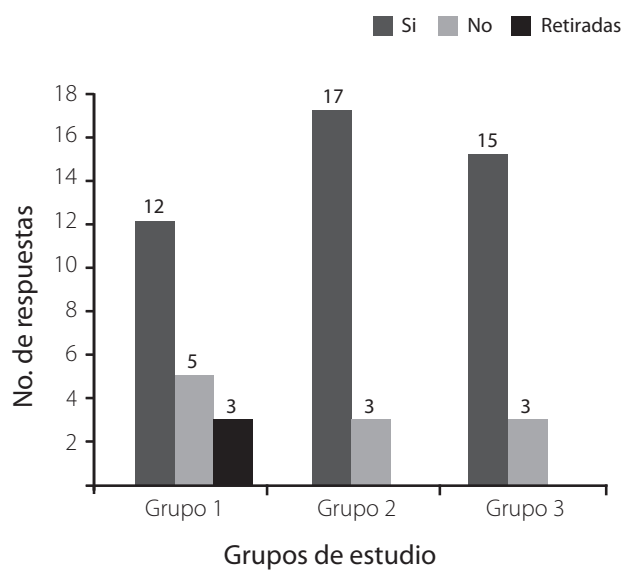

FIG. 2. Respuestas de las trabajadoras sexuales entrevistadas acerca de si realizan o no alguna otra actividad laboral además del trabajo sexual.

Algunas expresan deseos de continuar estudios o de conformar un hogar: "De aquí a 5 o 10 años me veo más feliz, escribiendo un libro, que exponga la realidad de la vida que llevan las trabajadoras sexuales." - "Me veo a futuro con una microempresa exitosa y siendo una persona de respeto" - "Me veo administrando un spa en forma honrada." - "Me gustaría tener una profesión, una casita propia para vivir con mis hijos." - "Me gustaría casarme y tener una familia." Otra dice sentirse satisfecha-"Me siento confortable así, tengo un novio, pero él no sabe a qué me dedico, pero si me llegara a casar con él, dejaría el trabajo sexual." Y finalmente, otra teme un futuro terrible: "Me veo viviendo a futuro en un asilo de locos, durmiendo en la 
calle, muerta o asesinada por algún enemigo en la calle. Yo ya no veo ninguna posibilidad para mi vida de algo diferente, pues la necesidad me llevó a caer en este trabajo, hace ya 30 años."

\section{Conflictos familiares}

Otra consulta que se le hizo a las entrevistadas fue la existencia o no de conflictos en su familia de origen; un ejemplo de las respuestas obtenidas es el de Marisol, de 35 años, quien refiere que su papá es un alcohólico que siempre los agredió, tuvo "pleitos constantes" con su mamá y hermanos: "cuando llegaba borracho, todos corríamos a escondernos para que nos nos pegara".

Tan importante como que dos tercios de ellas mencionan conflictos en sus hogares de origen, es el dato de que un tercio dice que no había conflictos en su hogar (Fig. 3).

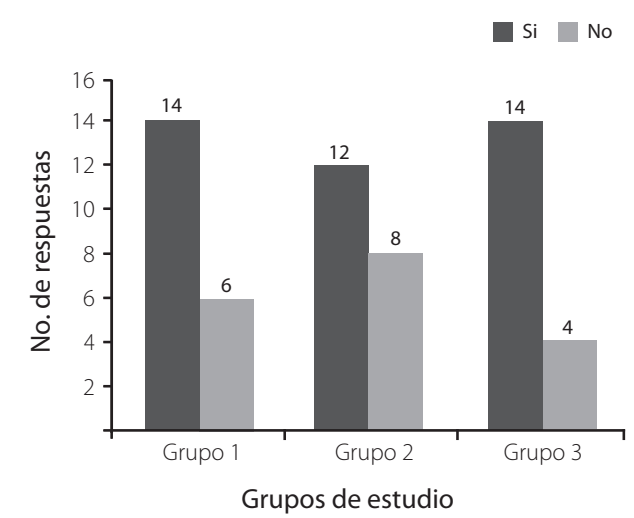

FIG. 3. Respuestas de las trabajadoras sexuales entrevistadas ante la pregunta: ¿En su familia de origen, se daban conflictos, peleas, divorcio?

\section{Su actualidad}

\section{Relación entre el trabajo y las creencias religiosas}

Las trabajadoras entrevistadas son todas cristianas (católicas la mayoría, seguidas de protestantes e incluyendo una mormona). La mayoría (58,6\%) afirma que no sienten un conflicto religioso por su trabajo (Fig. 4). Representativa de la mayoría es Karla, de 26 años y del grupo 2 , quien dice que las creencias religiosas se olvidan en el trabajo.

Estas son las declaraciones de la minoría que dice sufrir un conflicto: Sharon, de 31 años y también del grupo 2, refiere "...todos los días le pido a dios perdón y entendimiento, pero si esto que hago es malo, todavía es peor que mis hijas pasen hambre. Él sabe que es un trabajo que hago por necesidad".

También del grupo 2, Fernanda, de 28 años, dice "en la misa yo no comulgo porque trabajo en esto, y no puede confesarme porque si el padre se da cuenta de lo que hago me quita la ayuda que me dan en la iglesia. Pero todos los días le pido perdón a dios y le prendo velitas".

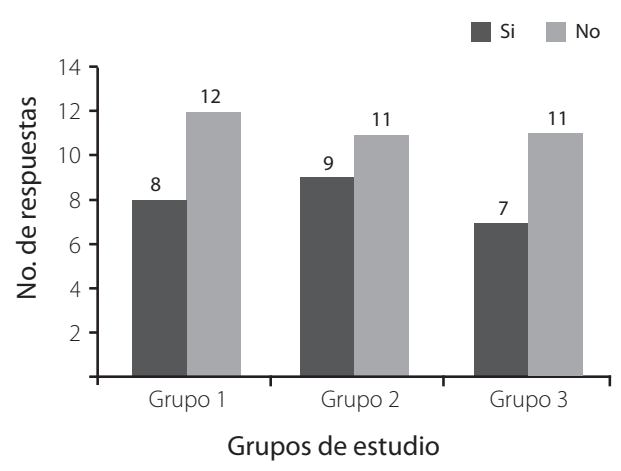

FIG. 4. Respuestas de las trabajadoras sexuales entrevistadas con respecto a si tienen o no conflictos por su trabajo y sus creencias religiosas.

\section{Protección contra enfermedades de transmisión sexual}

La gran mayoría afirma que se protegen de enfermedades de transmisión sexual y usan para ello el preservativo, además de que van a control médico. Solamente dos, del grupo uno, afirmaron que no usan protección, que han sufrido enfermedades y que actualmente están curadas (Cuadro 3).

\section{El medio social}

\section{Cómo debe ser una mujer}

Estos son ejemplos de respuestas ante la pregunta de cómo debe ser una mujer. Carla, 26 años: "Una mujer es un objeto sexual, un hombre es superior "; Kily, 25 años: "La sociedad es quien nos dice qué ser y jugamos esos papeles ", Marisol, 33 años: "Los medios de comunicación y la sociedad dicen que la mujer debe de ser señora en la casa, las que hagan lo contrario son zorras; el hombre haga lo que haga sigue siendo hombre, puede ser borracho y andar con cien mujeres y sigue siendo hombre, lo saludan igual "; Paola, 24 años: "La mujer es la que está en la casa, hace la comida, cuida a los hijos y está para satisfacer las necesidades del hombre, el hombre es el que trabaja y lleva la comida a la casa".

Erika, de 32 años, aportó un concepto más equitativo: "La mujer es la figura familiar encargada de la casa, es la persona que debe de ser querida, no debe de estar 


\section{CUADRO 3}

Respuestas de las entrevistadas a la pregunta ¿Cómo se protege de las enfermedades?

\begin{tabular}{lccc}
\hline Método de prevención & Grupo 1 & Grupo 2 & Grupo 3 \\
\hline Preservativo y control & 7 & 3 & 5 \\
médico regular & 8 & 16 & 11 \\
Sólo con preservativo & 2 & 0 & 0 \\
Sólo control médico & 2 & 0 & 0 \\
No se protege & 1 & 1 & 2 \\
NR & & & \\
\hline
\end{tabular}

estigmatizada ni ser criticada, el hombre es la cabeza del hogar sin dejar del lado a la mujer, que es un complemento".

\section{El papel del trabajo sexual femenino en la sociedad}

Evelyn dice que el trabajo sexual es necesario para la sociedad, ya que si no existiera, "...habrían más violaciones de niños y mujeres, pero con nosotras los hombres así se desahogan, y acá hemos tenido casos de hombres que nos piden actuar como niñas, si no tuvieran esta opción, podrían ir y hacerlo de verdad". Kilia dice, "Esta es la profesión más vieja y existe porque muchas mujeres no les dan a los hombres lo que quieren, o la esposa está embarazada, y entonces ellos nos buscan para desahogarse". Jenny: "Sin nosotras los matrimonios durarían menos, porque sin esta opción los hombres no aguantarían tanto tiempo casados con la misma mujer". Karina afirma que este trabajo es muy necesario para las mamás solteras, "...para mantenerse, pero para los hombres casados es un pasatiempo". Para Ivannia "Es una alternativa económica y para el hombre es necesario porque buscan un escape a la rutina."

\section{DISCUSIÓN}

\section{Quienes son}

"Las Cuarenta", documental de cine del director Víctor Vega (Centro Costarricense de Produción Cinematográfica, 1975) caracteriza a las trabajadoras sexuales de hace tres décadas en San José como mujeres asociadas con dificultades económicas, conflicto familiar, migración y baja escolaridad, al igual que prácticamente todos los demás autores tradicionales como Acuña et al. (1982).

Sobre esta conclusión, Schifter (1998) identificó dos errores metodológicos presentes en gran proporción de los estudios sobre trabajo sexual, en Costa Rica y en el resto del mundo:

1. Aún cuando declaren una visión objetiva y antipatriarcal, la mayoría de sicólogos, sociólogos y demás autores parten de la visión patriarcal de que el trabajo sexual es indeseable y diferente del resto de los trabajos. Este es el enfoque patriarcal subyacente.

2. Tras describir las características de las personas que realizan trabajo sexual, concluyen que esas características son las causas del trabajo sexual.

El enfoque patriarcal subyacente es un error porque el concepto del sexo como malo, la santidad del matrimonio y en general la moral sexual cristiana, islámica y de otras religiones patriarcales, son constructos ideológicos que carecen de bases biológicas (Barkow 2005).

La conclusión de que algunas características de una población automáticamente son causas de otras características viola los principios básicos del pensamiento científico y es injustificada porque la relación causa efecto solo puede evaluarse mediante experimentos formales; de lo contrario, se llega fácilmente a conclusiones erróneas (James y McCulloch 1990).

En el caso de las trabajadoras sexuales, las mismas características de dificultades económicas, conflicto familiar, migración y baja escolaridad se dan en millones de mujeres que nunca en su vida se llegan a dedicar al trabajo sexual: una "causa" que la mayoría de las veces no causa un efecto, no es una verdadera causa sino parte de un fenómeno mucho más complejo (James y McCulloch 1990).

En 1980 , solo el $46 \%$ de las trabajadoras provenían de ambientes urbanos (Acuña et al. 1982). Hoy son el $58 \%$, lo cual podría simplemente reflejar el hecho de que el porcentaje de población urbana del país ha ido en aumento (Costa Rica Ministerio de Economía 1998).

Al comparar los resultados de 1982 (Acuña et al. 1982) con los actuales, hay coincidencia en que la mayoría concluyó la educación primaria, pero pocas concluyeron la secundaria y muy pocas tienen nivel universitario. En esto, las trabajadoras sexuales son representativas de la población femenina general de Costa Rica, donde la tasa de deserción escolar es muy alta después de la escuela primaria (Espíndola y León 2002). En general, si tuvieran mayor educación, podrían subir en el escalafón que va desde las trabajadoras de calle poco educadas hasta las VIP más educadas. Además, el concluir sus estudios haría posible para ellas dejar el trabajo sexual para dedicarse a una profesión u oficio mejor recibidos por la sociedad patriarcal.

\section{Su historia}

Al igual que una buena parte de la población femenina costarricense proveniente de familias con bajo nivel 
económico, ellas recibieron poca ayuda directa con el trabajo escolar y consideran como un logro el haber concluido la educación primaria, y expresan a menudo su deseo de alcanzar un nivel más alto de escolarización, aunque pocas están estudiando. Este resultado es idéntico al de estudios previos (Acuña et al. 1982, Ortiz et al. 1998).

En 1980 un 68\% indicaron que habían crecido sin conflictos familiares Acuña et al. 1982), hoy solamente un tercio dijo lo mismo. Desconocemos si el aumento de mujeres que declaran haber tenido conflictos se debe a que existe un aumento real; a que hoy día se habla más abiertamente del tema; a que se ha ampliado el concepto de conflicto familiar a casi cualquier desacuerdo o incomodidad familiar; o a una combinación de todos estos elementos.

\section{Su actualidad}

El alto porcentaje de trabajadoras que sufrían sentimientos de culpa por violar preceptos de la religión patriarcal hallado en 1975 (Vega 1975) no se repitió en este estudio, posiblemente porque el dominio de las organizaciones religiosas sobre la población actual es menor .

La tendencia hacia el uso del preservativo que se detectó en la década de 1990 en relación con el temor al sida (Ortiz et al. 1998) ha evolucionado en 2007 a un uso generalizado y sistemático, con lo que estas trabajadoras están mejor protegidas que muchas mujeres que mantienen relaciones sexuales por amor (Boynton 2002). La presentación de las trabajadoras sexuales como transmisoras de enfermedades (ver Marín 2005) carece de fundamento y puede entenderse como fruto de la ideología patriarcal (Careaga y Cruz 2004).

\section{El medio social}

Usando la tasa de 2 trabajadoras por cada 1000 habitantes, (Acuña 1982), el San José actual (toda la ciudad, no solo la parte central que estudiamos acá) tendría un estimado de 3200 trabajadoras sexuales, aunque nuestra impresión tras el trabajo de campo es que la cantidad real es inferior a ese número, lo cual es un tema recomendado para un estudio futuro.

Pese a violar el precepto patriarcal de que la mujer cría a la familia y el hombre la provee de recursos (Ramírez et al. 2005), estas trabajadoras son típicas de la población general en que creen firmemente en ese precepto y sueñan con ser esposas. Esta creencia es coherente con la educación patriarcal que reciben todas las niñas en Costa Rica en las escuelas públicas, y en grado aún mayor, en muchas escuelas privadas de orientación religiosa (Ramírez et al. 2005).

La abundancia de hombres casados dentro de la clientela de las trabajadoras sexuales no ha variado entre los estudios de fines del siglo XX (Acuña et al. 1982) y el actual. Esta clientela estable de hombres que están casados y permanecen casados tras usar estos servicios, contradice la imagen de la trabajadora sexual como destructora de hogares (Marín 2005).

El argumento mencionado en una de las entrevistas, de que la disponibilidad de servicios sexuales pagados disminuye la presión sobre las esposas y el peligro de violación de menores y de mujeres, coincide con el hallazgo de que la disponibilidad de desfogues sexuales disminuye las violaciones (Diamond y Uchiyama 1999). La presentación en los medios de Costa Rica del "problema de Costa Rica como destino sexual" podría ser un caso semejante, dado que la cantidad de trabajadoras existentes solo podría atender a una fracción ínfima de los 2000000 de turistas que visitan el país anualmente (Costa Rica Tourism 2008).

\section{El papel de la UNED}

Desde la creación de la UNED en 1977, tanto su normativa como sus textos didácticos, están elaborados en un lenguaje patriarcal, en donde se omite en su mayoría, el género inclusivo. Un ejemplo es la unidad didáctica de Myriam Vázquez, reimpreso en el 2002, que afirma "...La Educación es un proceso continuo y permanente en un ser humano ... potencialmente dueño del mundo... de un hombre que exige ser considerado un hombre concreto, para realizar su 'ecuación personal'y el ideal humano, por hombres actuales en relación dialéctica, insertados en lo social..."

En contraste con esta realidad, la misión institucional establece un compromiso con la excelencia académica, el desarrollo de la cultura, la ciencia, el arte y los derechos humanos, para la construcción de una sociedad justa y una cultura de paz.

En relación con la población femenina joven como la estudiada por nosotros, la UNED podría apoyar más la entrada al Colegio de Educación a Distancia de mujeres pobres; así como programas educativos para adolescentes mediante la Dirección de Extensión. También se requiere actualizar en este sentido la praxis de la Dirección de Asuntos Estudiantiles, el Programa Institucional para la Equidad de Género y la Comisión de Valores. Se debería elaborar un documental para la televisión que cambie el enfoque patriarcal subyacente por una visión científica contemporánea y el Sistema de Estudios de Posgrado podría hacer más estudios en este campo, por ejemplo con historias de vida, ya que es una técnica que permitiría dar a las trabajadoras sexuales una voz desde la concepción de sus propios mundos y del ambiente en que se desarrollan. 


\section{AGRADECIMIENTOS}

Agradecemos a Mónica Chávez por su ayuda como asistente del proyecto; a las mujeres que compartieron sus historias y opiniones; a las salas de masajes y a la Fundación RAHAB que nos permitieron hacer entrevistas en sus locales; y a la Vicerrectoría de Investigación de la UNED por el apoyo financiero; así como a Katya Calderón, sin cuyo eficaz apoyo habría sido imposible concluir este estudio.

Iris Ramírez dedica este artículo: "A quienes en la oscuridad y en el silencio esperan encontrar sus propios rostros y oír sus propias voces... Son mujeres que como tú y como yo servimos a otros..."

\section{RESUMEN}

San José es la ciudad capital de Costa Rica, pequeño país centroamericano caracterizado por un mayor nivel educacional, mayor ingreso per capita y mayor nivel de vida que el resto de los países de la región. Un estudio hecho en 1975 indicó que las trabajadoras sexuales de la ciudad eran mujeres que habían elegido el trabajo sexual porque en él ganaban mucho más que en las demás opciones laborales que tenían, dado su nivel de escolaridad, en general limitado a la escuela primaria, y que cerca de la mitad no deseaban cambiar de actividad. Con el fin de conocer si la situación había cambiado casi cuatro décadas después, en 2008 entrevistamos a 78 mujeres que realizan trabajo sexual en la ciudad, con tarifas entre US\$3 y US\$100 la hora y edades 18 y 81 años. Las entrevistas se hicieron con base en un cuestionario; se pagó a las entrevistadas su tiempo y los datos se analizaron con el programa estadístico SPSS. La mayoría de estas trabajadoras son costarricenses de origen urbano, pocas tienen estudios más allá de la escuela primaria, y consideran que su principal logro en la vida fueron los años escolares aprobados pese a la poca ayuda que recibían en este campo de sus familias. Un tercio declaró haber tenido una niñez agradable, y la mayoría dicen no hallar conflicto entre su trabajo y las creencias religiosas, pues lo hacen para mantener a sus hijos, ya que son madres solteras. Creen además que su trabajo disminuye la violencia sexual. La mayoría usa el preservativo como protección y cree en la visión patriarcal de que la mujer cría a la familia y el hombre provee los recursos. Las universidades podrían ofrecerles a estas mujeres apoyo para concluir sus estudios, apoyando con becas y asesoría y haciendo más estudios para separar el mito de la realidad, por ejemplo con historias de vida, una técnica que permitiría dar a las trabajadoras sexuales una voz desde la concepción de sus propios mundos y del ambiente en que se desarrollan.

PALABRAS CLAVE

Educación, trabajo sexual, socialización, patriarcado, cultura, prostitución.

\section{REFERENCIAS}

Acuña, O., C. Denton \& F. Naranjo. 1982. La prostitución en San José. Un estudio socioeconómico de un problema costarricense. Universidad Nacional, Heredia, Costa Rica.

Barkow, J.H. (Ed.). 2005. Missing the Revolution: Darwinism for Social Scientists. Oxford University, Nueva York, Nueva York, EEUU.

Boynton, P.M. 2002. Life on the streets: the experiences of community researchers in a study of prostitution. Journal of Community \& Applied Social Psychology 12: 1-12.

Careaga, G. \& S. Cruz. 2004. Sexualidades diversas. Universidad Nacional Autónoma de México, México DF, México.

Costa Rica Tourism. 2008. Costa Rica Tourism Board rewards the two million tourist, San José, Costa Rica (Disponible en http://tourism.co.cr/costa-rica-tourism-news, consultado el 27 de diciembre de 2008).

Diamond, M. \& A. Uchiyama.1999. Pornography, rape, and sex crimes in Japan: Research advances and policy considerations. Journal of Law and Psychiatry 22:1-22.

Espíndola, E. \& A. León. 2002. La deserción escolar en América Latina: un tema prioritario para la agenda regional. Revista Iberoamericana de Educación 30: 39-62.

James, F.C. \& C.E. McCulloch. 1990. Multivariate analysis in ecology and systematics: Panacea or Pandora's box? Annual Review of Ecology and Systematics 21: 129-166.

Marín, H.J. 2005. La tierra del pecado, entre la quimera y el anhelo. Alma Mater, San José, Costa Rica.

Ministerio de Economía de Costa Rica. 1998. Estimaciones y proyecciones de población actualizadas a 1996. San José, Costa Rica.

Ortiz, M., A. Zamora, A. Rodríguez, L. Chacón \& A. Gutiérrez. 1998. Soy una mujer de ambiente. Universidad de Costa Rica, San José, Costa Rica.

Ramírez, S., I. Cascante, C. Eppelin \& G. Espinoza. 2005. Promoviendo la igualdad de oportunidades y la equidad de género para las niñas en edad escolar. Instituto Nacional de las Mujeres, San José, Costa Rica.

Schifter, J. 1998. Lila's House: Male Prostitution in Latin America Routledge, Nueva York, Nueva York, EEUU.

Vázquez, M. 2000. Principios y técnicas de educación para adultos. EUNED, San José, Costa Rica.

Vega, V. 1975. Las cuarenta. Centro Costarricense de Producción Cinematográfica, San José, Costa Rica (película documental)..

\section{Este artículo fue editado por Zaidett Barrientos}




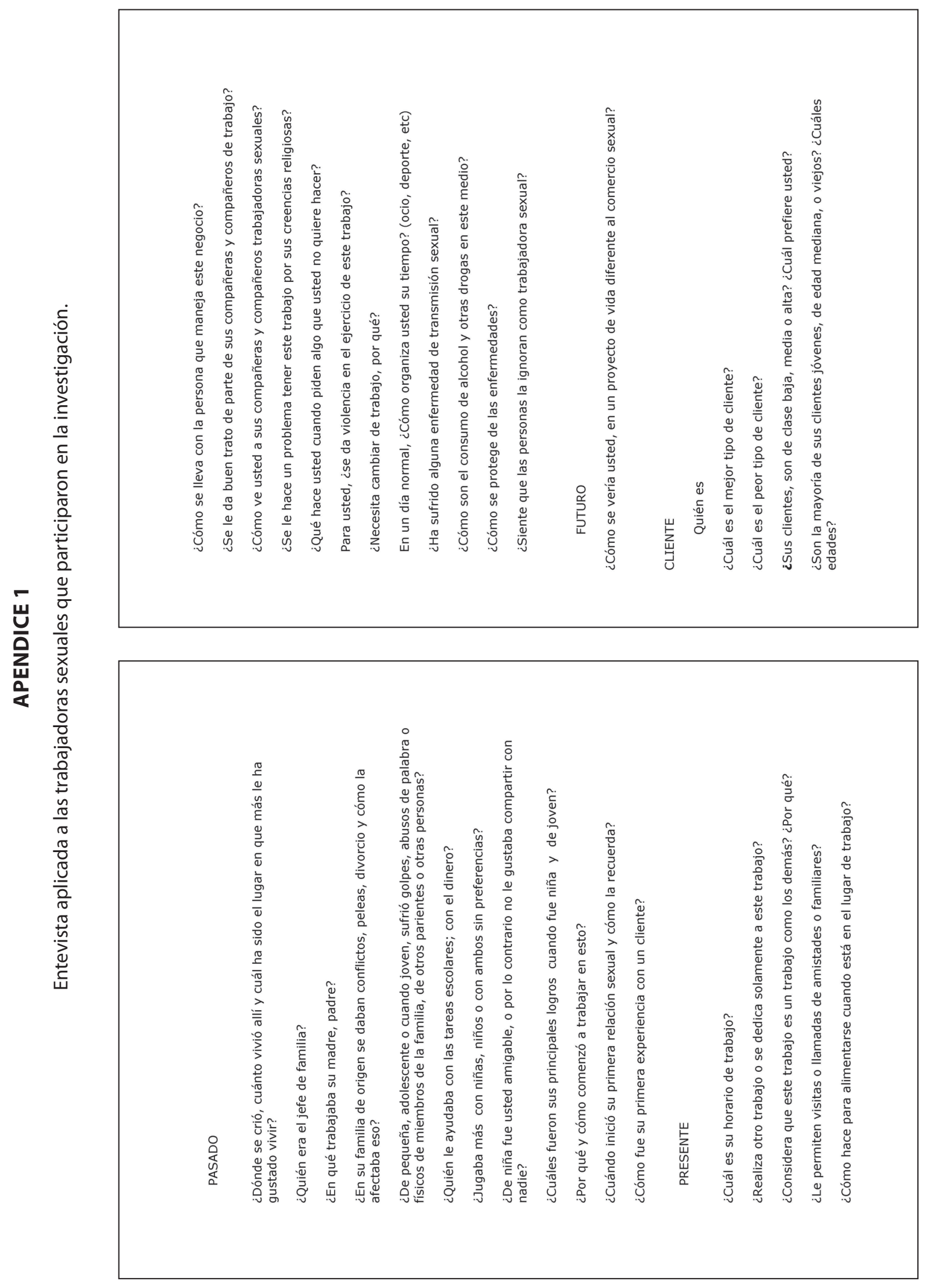



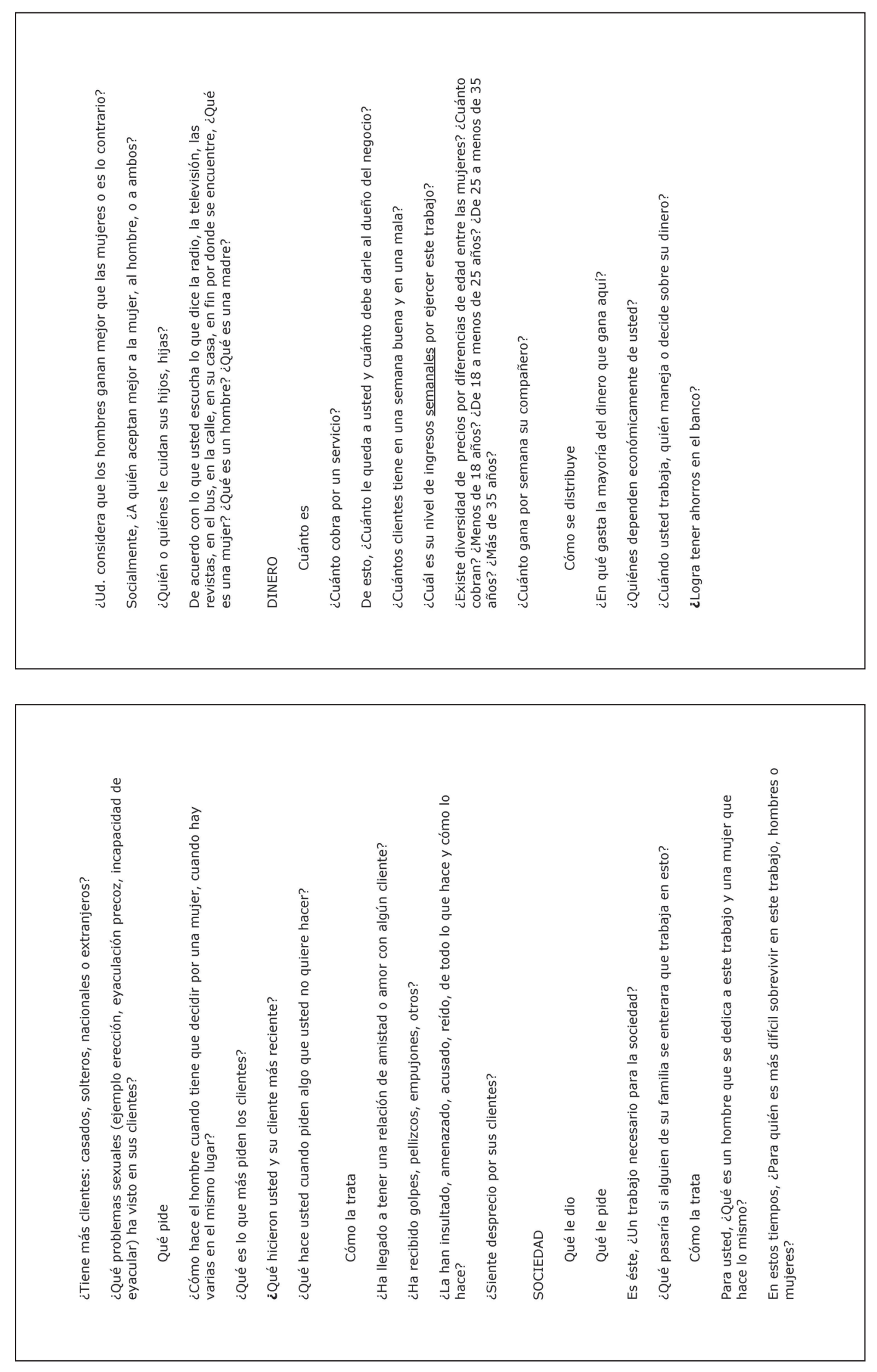